\title{
Serviços de referência virtual
}

Miguel Ángel Márdero Arellano

Mestre em Ciência da Informação

e-mail:miguel@ibict.br

\section{Resumo}

Analisar os serviços de referência virtual, seus padrões e novas tecnologias que têm modificado a prática tradicional realizada no balcão de referência das bibliotecas. São descritas as principais iniciativas norte-americanas e as características de seu funcionamento.

\section{Palavras-chave}

Serviços de referência virtual; Biblioteca digital; Tecnologias de informação; Referência digital; Bibliotecário de referência; Correio eletrônico.

\section{Virtual reference services}

\begin{abstract}
Analysis of virtual reference services, their standandars and new technologies that have changed the tradicional practice at the library's reference desk. Major American virtual reference services initiatives and their characteristics are described.
\end{abstract}

\section{Keywords}

Virtual reference services; Digital library; Information technology; Digital reference; Reference librarian; Electronic mail.
"For the Next Generation of Library Users"

(Morris City Library)

\section{INTRODUÇÃO}

Este trabalho tem por objetivo apresentar o estado atual dos serviços de referência virtual existentes no exterior, principalmente nos Estados Unidos, e identificar as características das principais iniciativas desenvolvidas até o momento. Tendo em vista o enorme crescimento da literatura sobre o assunto, este estudo pretende formular um conjunto de temas de pesquisa relacionados com um tipo de serviço específico das bibliotecas na Internet. O trabalho inclui conceituações, normas, dados e descrições desse tipo de serviços.

\section{DEFINIÇÃO DOS SERVIÇOS DE REFERÊNCIA VIRTUAL}

As bibliotecas digitais estão sendo concebidas como apenas coleções de materiais digitais que podem ser acessados por usuários remotos, mas os novos softwares desenhados para criar redes colaborativas de bibliotecas mostram que o conceito pode ser estendido para o acesso a serviços virtuais (Meola,1999). Há algum tempo surgiu, na lista de discussão bib-virtual do IBICT (http://www.cg. org.br/gtbv/lista.htm), uma dúvida sobre a qualidade dos serviços de referência que as bibliotecas disponibilizam na rede. Minha resposta na época foi que no Brasil não existiam serviços da natureza dos encontrados nos sites das principais bibliotecas americanas. Ainda no início de 2001, nenhuma das 184 bibliotecas brasileiras cadastradas pelo IBICT no GT de Bibliotecas Virtuais (http:// www.cg.org.br/gt/gtbv/alfabetica.htm) mantinha algum serviço de referência que funcionasse 24 horas e com distribuição seletiva do tipo de consultas.

Existem, no exterior, na rede, centenas de serviços de consulta que recebem grande volume de questões por dia. Serviços como Ask Jeeves (http://www.ask.com) e WebHelp (http://www.webhelp.com) são operados por empresas atendendo à demanda de clientes e seguindo os interesses financeiros de seus investidores. Por outro lado estão os serviços de referência digital "AskA" não comerciais financiados por centros de pesquisa e usados por estudantes, pais, educadores e outros para conectarse com profissionais experientes da área da biblioteconomia e serem guiados às melhores fontes de informação e especialistas de todas as áreas do conhecimento. Quando esse serviço é operado por uma 
biblioteca, tem como característica uma otimização na qualidade das respostas, no tipo de profissional encarregado e no público para quem ele está sendo orientado (Coffman, 2000). Os serviços de referência das bibliotecas com bibliotecários treinados usam e avaliam as ferramentas e os recursos na rede para a otimização dos resultados (Sullivan, 2001).

Atualmente muitos desses serviços estão reduzidos a consultas enviadas por correio eletrônico, telefone ou formulários na Web, consumindo tempo e exigindo um trabalho árduo de pesquisa. O longo processo resulta muitas vezes no comprometimento da troca efetiva de recursos e informações que permitam a colaboração entre diversos tipos serviços de referência.

Os serviços de referência virtual estão se tornando realidade, e são parte ativa na evolução dos serviços das bibliotecas na Internet. No início de 2001, teve lugar na Library of Congress o primeiro simpósio para discutir os conceitos e as implementações dos serviços de referência virtual (http://cweb.loc.gov/ala/digiref.html). Nele o professor David Lankes, da Syracuse University's School of Information Studies, apontou o status atual desse serviços. Ele citou um estudo no qual 97,3\% das bibliotecas universitárias americanas já possuem algum tipo de serviço de referência digital, definido como "mecanismo pelo qual as pessoas podem enviar perguntas e obter respostas através de e-mail, chat ou formato Web" (Saunders, 2001).

Uma demonstração do avanço do interesse na área dos serviços de referência eletrônica ocorreu na última reunião da American Libraries Association em 2000, em Chicago, onde havia apenas poucas bibliotecas com projetos de referência virtual. Este ano, na reunião de janeiro da ALA, eram mais de 65 bibliotecas usando o software LSSI (http:/ /www.lssi.com/virtual) para referência virtual e dezenas usando outros programas, tais como Live Person, eShare e HumanClick. Atualmente o assunto "referência virtual" está presente em muitos eventos da área nos Estados Unidos e é tema em fóruns especializados, tais como as listas de discussão Dig_Ref Listserver (http://vrd.org/ Dig_Ref/dig_ref.html) para pessoas e organizações interessadas no assunto, mantido pelo Virtual Reference Desk Projet, e na lista Live Reference Listserver (livereference@egroups.com). A preocupação atual desses pesquisadores está na sua institucionalização e na criação de padrões técnicos e de qualidade comuns e, também, no desenvolvimento de softwares que possam ser usados tanto nos serviços de referência em tempo real como nos de referência assíncronos.

\section{CARACTERÍSTICAS DOS SERVIÇOS}

Os serviços de referência virtual via correio eletrônico surgiram nos Estados Unidos no final da década de 1980, ao mesmo tempo em que as bibliotecas começaram a colocar seus catálogos na Internet. Alguns desses catálogos permitiam que os usuários remotos submetessem suas perguntas através de links que possibilitavam o pedido de consulta de um documento. Assim como os serviços AskA-Scientist e Ask-A-Volcanologist, que recebem perguntas por e-mail específicas sobre uma área da ciência, o serviço Ask a Librarian (Pergunte a um bibliotecário) existe na Internet há alguns anos e é fácil de ser acessado pelos motores de busca quando alguém faz uma pergunta. São os departamentos de referência das bibliotecas que criam esse serviço como um link na home page da biblioteca, aumentando consideravelmente o número de consultas. Muitas vezes o serviço que era apenas dirigido a uma específica comunidade acadêmica aumentou com o recebimento de mensagens provenientes de várias partes do mundo. Serviços como Google, Fast e Altavista são indicados como os promotores desse tipo de explosão no uso da referência virtual.

$\mathrm{Na}$ Internet podem ser encontradas bibliotecas que oferecem serviços de referência no tempo real via acesso à base de dados, telefone, e-mail, formulário na Web, videoconferência, "Internet chat", páginas de FAQs ou Mural.

Os serviços de referência chamados também de Ask-AnExpert (AskA) são o oposto das páginas estáticas e têm como objetivo desenvolver atitudes necessárias para a pesquisa nos seus usuários (Kasowitz, 1998). As pessoas que servem na referência digital são especialistas (voluntários, orientadores, bibliotecários e outros); podem ser chamadas de especialistas da informação porque realizam o trabalho similar àquele já estabelecido na biblioteca física. Alguns desses serviços têm um público específico, como o KidsConnect, para estudantes de primeiro grau, outros direcionados aos professores desses alunos, como o AskERIC, e outros oferecem informação específica sobre um assunto para todo tipo de público, como o MAD Scientist Network. Atualmente esses serviços de referência oferecem dois tipos de especialidade, informação sobre temas práticos e informação de referência e instrutiva.

\section{Tipos de Suporte e Funcionamento}

Diversas bibliotecas estão realizando testagem de serviços de mensagens de e-mail instantâneos chamados de "Live Help", os quais provêm apoio na pesquisa e navegação para os usuários das bibliotecas na Web. Os programas devem ser avaliados como projetos pilotos para testar sua 
eficiência na resposta imediata e se eles respondem às necessidades do tipo de informação que é requerida. Um exemplo é o NCI Information Specialists, que dá apoio ao serviço "Live Help" do National Cancer Institute com informações sobre o câncer, não dá consultas, mas dá assistência às pessoas que navegam no seu site. A home page com o piloto do serviço está no endereço http:// cancer.gov/livehelp/beta/phase2.html. Os promotores do projeto mantêm os usuários atualizados com as mudanças no serviço e aceitam as sugestões dos participantes no período de testes.

O serviço de referência via telefone tem sido usado tradicionalmente pelas bibliotecas como a melhor forma de oferecer seus serviços para usuários remotos, mas o custo pode ser considerável ao se tratar de chamadas de longa distância. $\mathrm{O}$ serviço de referência via correio eletrônico possui as mesmas vantagens, como também permite o envio e recebimento da informação no horário mais conveniente para o usuário. Outras vantagens do correio eletrônico usado no serviço de referência são o anonimato e a permissão da impressão de todo o processo de consulta e do resultado da pesquisa.

Por sua parte, a videoteleconferência de referência, que surgiu no Interactive Reference Service da University of California (http://www.ala.org/acrl/paperhtm/a10.html), tem demonstrado nos últimos anos a necessidade de novas regras de etiqueta para a comunicação entre usuários e o pessoal encarregado; o suporte tecnológico efetivo deste último grupo é fundamental para o funcionamento do serviço em tempo real. Atualmente, o uso da tecnologia de teleconferência é considerado fundamental para o desenvolvimento de coleções e serviços digitais (Lessik, 1997).

Uma característica que se destaca nesses projetos é a dos bibliotecários de referência on-line que estão se especializando no uso das tecnologias e das obras de referência existentes na rede. $\mathrm{O}$ perfil e as tarefas do servidor ou novo bibliotecário de referência surgem caraterizando um tipo de profissional que não mais realiza seu trabalho usando apenas obras em papel, opacs e bases em cd-rom. Um exemplo é Gary D. Price, da Virginia Campus Library, na George Washington University, que preparou um site contendo links para as obras de consulta mais usadas na área de referência (http://gwu.edu/ gprice/ handbook.htm) e para algumas bases de dados "escondidas" na rede (http://gwis2.circ.gwu.edu/ gprice/ direct.htm). Essas iniciativas são comuns entre os bibliotecários responsáveis pelos "sites das bibliotecas". A Iowa State University tem um real-time librarian, Gary McKiernan, especializado em referência digital em tempo real "_LiveRef(sm)”. Ele tem identificado bibliotecas que oferecem serviços desse tipo (http:// www.public.iastate.edu/ CYBERSTACKS/LiveRef.htm). Também, é a página de um bibliotecário na Internet a que possui a mais completa bibliografia sobre serviços de referência (Sloan, 2000).

Segundo Kasowitz (1998), o papel do especialista da informação na referência não é apenas o de "oferecer respostas", mas sim preparar usuários e estudantes de todos os níveis para resolver efetivamente suas necessidades de informação e ajudar a formar um pensamento crítico de suas fontes para pesquisa.

\section{Softwares}

Segundo Zick (2000), os bibliotecários estão muito preocupados com o futuro da sua profissão, pensando que podem ser substituídos pelos "sistemas inteligentes". O profissional da informação precisa trabalhar junto a esses sistemas inteligentes procurando as mesmas metas: dinamizar colaboração entre agentes, enriquecer o usuário e padronizar a informação para um tipo específico de público. Nos últimos anos, a interoperabilidade dos softwares para os serviços de referência virtual está sendo discutida, devido principalmente ao crescimento de novos sistemas de correio eletrônico e de softwares interativos e em tempo real. Alguns dos softwares mais usados: Humanclic, LivePerson, AOL Instant Messenger, AOL AIM Express, Camden, LiveAssistance, Webmaster. A Library of Congress (1998) menciona os seguintes softwares como a nova geração da referência on-line: EBSCO's Collectanea (http://www.collectanea.com), ISI's Web of Sience e os da OCLC (Online Computer Libray Center): Webline, FirstSearch, SiteSearch e FirstSearch Electronic Collections Online (http://www.oclc.org/oclc/ promo/7775os/reference.htm).

Os softwares interativos usados para os chamados chats ou "bate-papo" e os "Instant Messaging", apesar de serem muito populares na Internet, não são muito usados nas bibliotecas devido às complicações que apresentam tanto sua configuração como o seu funcionamento. Atualmente com os novos "Java browsers" que permitem que os programas de chat já estejam integrados nas páginas web, eles estão se convertendo no intermediário entre a referência via correio eletrônico e a videoconferência (Meola, 1999). Softwares tais como o NetMeeting e o NetShow da Microsoft, o Daedalus, Group Wise, Web Publisher, Norton Connect Net e o Reilly WebBoard estão sendo usados por bibliotecas escolares americanas por serem os mais conhecidos entre os alunos. Autores como Morgan (1999) acreditam que a videoconferência pode ser usada com sucesso na referência digital, por ser uma 
tecnologia e uma mídia de comunicação alternativa. Os bibliotecários precisam apenas obter o hardware necessário e usar programas como "CU-SeeMe", permitindo o acesso a grupos restritos de usuários, possibilitando também a educação a distância. São várias as experiências com esse tipo de software na Rede (Folger, 1997)

\section{Normas e Políticas}

Por muitos anos os serviços de referência têm sido conduzidos informalmente, apesar de existirem normas e políticas para eles nas bibliotecas, mas nenhuma aplicada a suas versões virtuais (Sloan, 1988). Segundo Bernie Sloan, o desenvolvimento de políticas e padrões para essa área deve considerar sua administração, os usuários, o pessoal, a infra-estrutura para os serviços, os custos e avaliações.

Os serviços de referência via telefone têm seguido um mesmo tipo de política para seu funcionamento: resolver as necessidades de informação do solicitante no tempo que dura a ligação, que, na maioria das vezes, não é cumprida devido ao excesso da demanda e ao tempo limitado do serviço. Já o serviço de referência via correio eletrônico, desde seu início, seguiu dois padrões estabelecidos em 1982, o padrão RFC 821 Simple Mail Transfer Protocol e o RFC 822 Standard for the Format of ARPA Internet Text Messages, os quais falam da extensão apropriada das mensagens e do conteúdo permitido (Philip, 1997).

Com o crescimento dos serviços de referência implementados pelas bibliotecas no exterior, observa-se que a ênfase no desenvolvimento de medidas e padrões não o está acompanhando. As bibliotecas digitais estão começando a criar uma série de padrões para efetivar a qualidade dos seus serviços. Segundo McClure (2001), é necessário entender em primeiro lugar a natureza da qualidade do serviço de referência, a qual está imersa no seu papel de guia para usuários de recursos de informação. A avaliação desses serviços é fundamental para seu planejamento (pessoal, equipamento, custos etc.) e principalmente para garantir que o usuário terá suas necessidades de informação cobertas.

A criação de políticas para envio de e-mail é um dos primeiros passos para o estabelecimento de um serviço de referência digital. Um exemplo de serviço de referência virtual foi o criado em Drexel University pela W. W. Hagerty Library (http://www.library.drexel.edu/services/ refquestion.html), onde estão listados os especialistas segundo a área do conhecimento com seus endereços de e-mail para que, dessa forma, os estudantes e professores possam contatá-los diretamente. Também, a University of Illinois at Urabana Champaign mantém um site com links para mais de 90 bibliotecas universitárias com serviços de Ask A Reference question, todos eles com suas próprias políticas para envio de e-mails (Sloan, 1997).

\section{Iniciativas}

$O$ primeiro serviço de referência on-line no mundo a funcionar 24 horas por dia foi o da North Carolina State University's Virtual Reference Service (http://www.lib. ncsu.edu/libref/). Já o primeiro projeto cooperativo de referência entre bibliotecas universitárias foi no estado americano de Illinois, envolvendo 14 bibliotecas: The Alliance Library Project. $O$ projeto tem um plano de avaliação em que são discutidos os métodos e técnicas usados procurando uma nova aproximação para o funcionamento da referência on-line (http://www.rsa.lib.il.us/ready/ about.htm). Também, nele foi incrementado um plano de marketing que aponta as estratégias a serem analisadas dentro de um serviço de referência on-line.

Cornell University Libraries também prove um serviço de referência "after hours" fundamentado no uso de tecnologia de referência virtual ao vivo (http:// campusgw.library.cornell.edu/services/ask.html). A instituição tem desenvolvido um plano para 2000-2002 que inclui a idéia de que os usuários deverão ter acesso 24 horas por dia, nos sete dias da semana, a um serviço de referência com qualidade, de qualquer lugar onde estiverem. Nos anos 90, a Cornell University Libraries iniciou a referência via e-mail, com tutoriais on-line e videoconferências; desde o ano passado oferece LiveHelp usando o software LivePerson, o qual requer apenas uma conexão na rede e um browser. Esse serviço está sendo usado preferentemente por estudantes de graduação.

A Library of Congress desenvolveu um parceria colaborativa chamada de "Collaborative Digital Reference Service” (CDRS) (http://www.loc.gov/rr/ digiref) junto com outras bibliotecas sem nenhum custo, apenas com o compromisso de disponibilizar parte do pessoal de cada biblioteca para responder pelo menos a 10 perguntas por semana e também permitindo o envio de suas perguntas para o CDRS. O propósito do CDRS é prover um serviço especializado de referência para usuários em qualquer lugar, a qualquer hora e através de uma rede internacional de bibliotecas digitais. A infra-estrutura para suportar o sistema inclui acordos de operacionalidade, descrições dos serviços e do software usado no acompanhamento e gerenciamento da rotina de perguntas e respostas, assim como a Library of Congress está construindo bases de dados com o perfil dos usuários e bases para as perguntas e respostas mais comuns (em parceria com a OCLC - Online Computer Library Center). 
Para a Library of Congress já é reconhecida a contribuição que o CDRS vem oferecendo no aumento da relevância das bibliotecas nas comunidades locais.

A iniciativa da Library of Congress, que atualmente encontra-se na Fase 3 com mais de 50 bibliotecas participantes, responde em realidade a uma necessidade de usuários das bibliotecas na Internet de todo o mundo que desejam um serviço de referência apurado e rápido. Isto já está sendo uma realidade para muitas bibliotecas de língua inglesa que participam do consórcio EARL (UK Public Library E-ref consortium) (http://www.earl.org.uk/ ask), que inclui um "request management system" com o qual qualquer pessoa nos Estados Unidos que envia uma consulta às três horas da madrugada, quando a biblioteca mais próxima está fechada, pode ter sua pergunta respondida por algum membro do consórcio na Austrália no tempo real (Ormes, 1998).

Os consórcios de bibliotecas têm-se afirmado como uma via para o estabelecimento de serviços de referência mais ágeis. Um exemplo é o projeto 24/7 Reference Project (24 horas e sete dias) do Metropolitan Cooperative Library System, patrocinado pelo Federal LSTA e administrado pela California State Library (http:// www.247ref.org). O software Webline é acessado via Web, usando aplicativos em java, precisando-se apenas de uma conexão na rede um browser IE 5.0 e pelo menos $64 \mathrm{Mb}$ de memória e um processador Pentium de $200 \mathrm{Mhz}$ ou mais veloz. O projeto conta já com mais de 40 bibliotecas participantes e uma rede de especialista por área, com construção de FAQs e testagem de cada parte integrante do processo de referência. $O$ resultado de um dos seus testes ressaltou a necessidade de incrementar o grau de conhecimentos de informática dos bibliotecários e o estágio iniciante no qual se encontra a referência por meio de chats. A Santa Monica Public Library (http:// www.smpl.org/library) é um exemplo de biblioteca participante de consórcio que vem realizando estudos sobre como ajustar seu serviço de referência interativo e em tempo real às necessidades dos seus usuários. Ela participa também do projeto CDRS e continua realizando o trabalho de avaliação da entrevista de referência via e-mail, da qual é uma das pioneiras desde 1989.

Um resultado de outro consórcio é o Virtual Reference Desk (http://vrd.org/about.html). Trata-se de um projeto dedicado ao melhoramento dos serviços de referência e à criação e operação de serviços de informação baseados na Internet com mediação humana. O projeto, patrocinado pelo United States Department of Education com apoio da White House Office of Science and Technology Policy, foi lançado como um serviço de referência cooperativo em janeiro de
2000, com 18 serviços de AskA e 28 profissionais da informação voluntários. No início de 2001, já eram 54 voluntários e vários novos serviços de AskA foram acrescentados; praticamente são bibliotecas públicas, universitárias e escolares, bem como estudantes de biblioteconomia que estão fazendo estágios dentro do projeto.

O projeto oferece aos participantes suporte técnico para os usuários e administradores do sistema de cada instituição onde estiver sendo usado o software. O AskA Consortium é o conjunto de organizações que fazem parte do projeto e realiza estudos sobre os padrões de interoperabilidade, metadados e outros aspectos do serviço "AskA" (http:// www.vrd.org/Tech/QuIP/index.html). Fazem parte do Consórcio: Ask A MAD Scientist, AskERIC, Internet Public Library, Morris County Public Library (NJ), National Museum of American Art, Library of Congress's American Memory Center for School Safety e Eisenhower National Clearinghouse for Mathematics and Science Education.

O projeto VRD, além de conectar as bibliotecas com os serviços de AskA (AskA Volcanologist, Ask Dr. Math, Ask Shamu, AskERIC, Ask A MAD e outros), promove conferências anuais (VRD, 2000 Conference Proceedings) e edita publicações sobre o tópico e um manual AskA Starter Kit (http://vrd.org/training.html). Um dos primeiros manuais para referência virtual foi elaborado na Library Systems \& Services LSSI (http://www.lssi.com), por Anne Lipow e Steve Coffman; sua versão preliminar foi lançada na última reunião da ALA. O título do manual é Establishing a Virtual Reference Service: VRD Training Manual (http://www.library-solutions.com/vrd.html). Foi desenvolvido inicialmente para o Bay Area Library Project, que usa software para referência Virtual da LSSI, mas atualmente é usado por mais de 70 bibliotecas americanas. Ele contém diretrizes para a organização de qualquer tipo de serviço de referência baseado no contato direto via web usando esse software. Eis alguns tópicos do manual: vista preliminar do software, planejamento de serviços de referência on-line, serviços, políticas e metodologias, treinamento, design e conteúdo das telas e exercícios sobre como usar o software. Anne Lipow é pioneira na área, e seus artigos abordam sempre temas associados à relação usuário-bibliotecário de referência e à importância de seu papel na era digital (Lipow, 1999). A proposta é que o manual seja atualizado três vezes por ano, para adaptações a mudanças e necessidades dos usuários.

Por último, cabe citar o "AskEric" (http://www. askeric.org), um serviço de referência virtual desenvolvido pela ERIC Clearinghouse on Information \& Technology/ Information Institute of Syracuse, orientado para a comunidade acadêmica, com informações sobre 
educação para professores, estudantes, orientadores, administradores e pais de família. Conta com uma coleção de 3 mil recursos de informação recopilados pelo seu corpo de especialistas, organizados alfabeticamente (http:// ericir.syr.edu/Search/topicsA-Z.html). Esses profissionais também dão assistência por meio do Ask na ERIC Expert Service (ver anexos 1 e 2 ).

\section{Conclusão}

O avanço das tecnologias da informação e seu impacto em todas as áreas da sociedade têm produzido a necessidade de se obter informação útil e relevante via fontes de informação especializadas, aquelas reconhecidas pelos seus métodos para encontrar, selecionar e difundir informação. As bibliotecas estão sendo designadas para cumprir essa função, por serem sistemas de informação que facilitam o acesso simples e efetivo a recursos de informação on-line.

Desde seu surgimento, os serviços de referência têm sido considerados como uma função de ensino para o uso racional dos recursos das bibliotecas (Rader, 2000). Iniciar um serviço de referência virtual requer levar em consideração como ele se enquadra dentro da missão da biblioteca e na cultura institucional, especificamente sua aceitação tanto pela gerência como pelo pessoal, e a possibilidade de contar com a infra-estrutura tecnológica apropriada para o projeto. Existe atualmente a necessidade, reconhecida dentro das diferentes iniciativas, de padrões que permitam a interoperabilidade dos serviços e do contínuo trabalho de testagem dos diferentes softwares que estão sendo usados pelas bibliotecas. Pode-se concluir que um primeiro exame das novas tecnologias aplicadas ao serviço de referência aponta para uma futura reprodução, quase idêntica em tempo real, da relação bibliotecáriousuário no balcão de referência das bibliotecas tradicionais.

\section{REFERÊNCIAS BIBLIOGRÁFICAS}

BRY, Lynn. Setting up an Ask-an-Expert Service. Washington : MAD Scientist Network, 1997. Disponível em: < http://www.madsci.org/ ask_expert/index.html>

COFFMAN, Steve; McGLAMERY, Susan. The librarian and Mr. Jeeves. American Libraries, May, 2000, p. 66-69. Disponível em: <http:/ /www.247ref.org/jeeves.htm>

FOLGER, Kathleen. The virtual librarian: using desktop videoconferencing to provide interactive assistance. In: ASSOCIATION OF COLLEGE AND RESEARCH LIBRARIES NATIONAL CONFERENCE PAPERS, 1997. [S. 1.] : American Library Association, 1997. Disponível em: < http://www.ala.org/acrl/papers.html>

KASOWITZ, Abby S. Guidelines for information specialists of K-12 Digital Reference Services. Virtual Reference Desk Publications, Oct. 1998. Disponível em: <http://vrd.org/training/guide.htm>
LESSICK, Susana; KJAER, Kathryn; CLANCY, Steve. Interactive Reference Service (IRS) at UC Irvine: expanding reference service beyond the reference desk. In: ACRL CONFERENCE, 1997. Disponível em: <http://www.ala.org/acrl/paperhtm/a10.html>

LIBRARY OF CONGRESS (United States). Reference service in a digital age. In: PROCEEDINGS OF THE INSTITUTE, 1998, Washington, DC. Washington, DC, 1998. Disponível em: <http:// lcweb.loc.gov/rr/digiref/>

LIPOW, Anne G. Serving the remote user: reference service in the digital environment. In: AUSTRALIAN LIBRARY AND INFORMATION ASSOCIATION CONFERENCE, 1999. Disponível em: <http://www.csu.edu.au/special/online99/proceedings99/200.htm>

McCLURE, Charles R.; LANKES, R. David. Assessing quality in digital reference services: a research prospectus. [S. 1.] : Information Institute of Syracuse, 2001. Dublin : OCLC, 2001. Disponível em: <http:// quartz.syr.edu/quality/Overview.htm $>$

MEOLA, Marc, STORMONT, Sam. Real-time reference service for the remote user: from the telephone and electronic mail to Internet chat, instant messaging, and collaborative software. Reference Librarian, n. 67/68, p. 29-40. 1999.

MORGAN, Eric Lease: see you see a librarian final report 1999. Disponível em: < http://sunsite.berkeley.edu/ emorgan/see-a-librarian/>

ORMES, Sarah. Public librries corner: ask a librarian. 1998. Disponível em: < http://www.ariadne.ac.uk/issue13/public-libraries/ > Atualização: dez. 1998.

PHILIP, Brenda. Mayhelpyou@theelectronicreferencedesk? An examination of the past, present and future of electronic mail reference service. [S. 1.] ; School of Library and Information Studies University of Alberta, 1997. Dísponível em: http://hollyhock.slis.ualberta.ca/598/ brenda/emailref.htm>

Hannelore B. Information literacy in the reference environment: preapring for the future. New Technologies and Reference, n. 71, p. 25-33, 2000.

SAUNDERS, Laverna. Building the virtual reference desk. In: SYMPOSIUM BUILDING THE VIRTUAL REFERENCE DESK IN A 24/7 WORLD, 2001, Washingto, DC. [S. 1. : s. n.], 2001. Disponível em: <http://www.infotoday.com/it/mar01/saunders.htm>

SLOAN, Bernie. Digital Reference Services: A Bibliography. Graduate School of Library and Information Science University of Illinois at Urbana-Champaign. November 7, 2000. URL: http://www.lis.uiuc.edu/ $\sim$ b-sloan/digiref.html

SLOAN, Bernie. Service Perspective for the Digital Library: Remote Reference Services. Graduate School of Library and Information Science University of Illinois at Urbana-Champaign. December 16, 1997. URL: http://www.lis.uiuc.edu/ b-sloan/e-ref.html

SLOAN, Bernie. Electronic Reference Services: Some Suggested Guidelines. Reference and User Services Quarterly 38:77-81. Summer 1998. Disponível em: < http://www.lis.uiuc.edu/ b-sloan/guide.html>

SULLIVAN, Danny. How Search Engine Rank Web Pages. 2001 URL: $<$ http://www.searchenginewatch.com/webmasters/rank.html>

VRD 2000 CONFERENCE PROCEEDINGS. URL: < http://vrd.org/ conferences/VRD2000/proceedings/index.html>

ZICK, Laura. The Work of Information Mediators: A Comparison of Librarians and Intelligent Softaware Agents. First Monday, 5. May 2000. URL: <http://firstmonday.org/issues/issue5_5/zick/index.html> 


\section{Anexo 1}

Serviços AskA

Ask A+ Locator

http://www.vrd.org/locator/index.html

* Internet Public Library Ask-A-Question Service

http://www.ipl.org/ref/QUE/

* Pitsco's List of Ask-an-Expert Services

http://www.askanexpert.com/category.htm

* All Experts

http://www.allexperts.com/

* ExpertCentral - A Breakthrough Way to Get the Answers You Need from Real Experts http://www.expertcentral.com

* Ask an Expert - Center for Research on Evaluation, Standards, and Student Testing (CRESST) http://cresst96.cse.ucla.edu/CRESST/pages/expert.htm

* The MAD Scientist Network

http://madsci.wustl.edu/

* Ask Dr. Math

http://forum.swarthmore.edu/dr.math/

* Ask Dr. Freemath

http://ois.unomaha.edu/drfreemath/

* Ask A Geologist

http://walrus.wr.usgs.gov/docs/ask-a-ge.html

* Ask The Space Scientist

http://image.gsfc.nasa.gov/poetry//ask/askmag.html 
Anexo 2

Serviços de Referências em Chat

Austin Community College Learning Resource Services Service:

Software: HumanClick

URL: http://www2.austin.cc.tx.us./JRB/opcserv1.htm\#REAL

Bowling Green State University, Jerome Library Reference Services Service

Software: HumanClick

URL: http://www.bgsu.edu/colleges/library/infosrv/hc.html

Broward Community College, University/College Library Service

Software: HumanClick

URL: http://exodus.broward.cc.fl.us/test/ask.htm

Canisius College Library Service

Software: HumanClick

URL: http://www.canisius.edu/canhp/canlib/humanclick.html

Carnegie Mellon University Libraries Service

Software: LivePerson

URL: http://www.library.cmu.edu/

Cornell University Library Service

Software: LivePerson

URL: http://www.library.cornell.edu/okuref/quest.html

Eastern New Mexico University, Golden Library Service

Software: HumanClick

URL: http://www.enmu.edu/ woodm/refchat.html

Georgia Institute of Technology, Library and Information Center Service

Software: AOL's Instant Messenger

URL: http://www.library.gatech.edu/reference.htm

Liberty University, External Degree Library Services Service

Software: AOL's Instant Messenger

URL: http://www.liberty.edu/resources/library/edp/index.html

Michigan State University's Main Library Service

Software: Netscape Chat

URL: http://www.lib.msu.edu/services/chat.html

North Carolina State University Libraries Service

Software: Netscape Chat

URL: http://www.lib.ncsu.edu/risd/libref/chat.html

Northwestern University of Louisiana, Watson Library Service

Software: Anexa.com Communities

URL: http://vic.nsula.edu/watson library/chat.html

Pepperdine University Libraries Service

Software: HumanClick

URL: http://rigel.pepperdine.edu/ 
Plattsburgh State University, Benjamin F. Feinberg Library Service

Software: AOL's Instant Messenger

URL: http://www2.plattsburgh.edu/acadvp/libinfo/library/iref.html

Rensselaer Polytechnic Institute, Rensselaer Libraries \& Information Services Service

Software: HumanClick

URL: http://www.rpi.edu/library/information/contact.html

SUNY College of Agriculture and Technology at Morrisville Library Service

Software: AOL's Instant Messenger

URL: http://www.morrisville.edu/library/talk.html

Temple University Libraries Service

Software: Camden

URL: http://www.library.temple.edu/ref/ask_us.htm

University Libraries, University at Buffalo Service

Software: AOL Instant Messenger and AOL AIM Express

URL: http://ublib.buffalo.edu/libraries/help/refchat.html

University of Chicago Library, Business and Economics Resource Center Service

Software: LiveAssistance

URL: http://www.lib.uchicago.edu/e/busecon/asklibrarian.html

Contact: see http://www.lib.uchicago.edu/e/busecon/aboutask.html

University of Edinburgh Library Service

Software:

URL: http://www.remote.lib.ed.ac.uk/

University of Illinois at Chicago, Library of the Health Sciences Peoria Service:

Software: HumanClick

URL: http://www.uic.edu/depts/lib/lhsp/services/helpdesk.shtml

University of Leicester, University Library, Distance Learning Unit Service

Software: HumanClick

URL: http://www.le.ac.uk/li/distance/enquiry/chat.html

University of North Texas Libraries Service

Software: ConferenceRoom by Webmaster

URL: http://www.library.unt.edu/chatroom/default.htm

University of Pennsylvania, Lippincott Library Service

Software: LivePerson

URL: http://www.library.upenn.edu/lippincott/askoption.html

University of South Florida, Tampa Campus Library

Florida Distance Learning Reference \& Referral Center Service

Software: ConferenceRoom by Webmaster

URL: http://www.rrc.usf.edu/chat/index.html

University of South Florida Virtual Library

Software: ConferenceRoom by Webmaster

URL: http://www.lib.usf.edu/virtual/chat/index.html

Virginia Tech Library

Software: LivePerson

URL: http://www.lib.vt.edu/ (from middle October 2000) 